\title{
CIENCIA, ÉTICA Y POLÍTICA: LA BIOÉTICA COMO CAMINO PARA LA TRANSFORMACIÓN DE LA PRAXIS CIENTÍFICA ${ }^{1}$
}

\author{
Ximena González Broquen²
}

Resumen: La "ciencia" se encuentra hoy en día en una situación paradójica: los fundamentos de la actividad científica y de las interrelaciones entre ciencia, técnica, industria, sociedad y Estado son fuertemente cuestionados y criticados, al mismo tiempo que sigue prevaleciendo mundialmente la idea de que la ciencia constituye el único motor para el desarrollo humano. Ahí es donde surge la idea de bioética como conocimiento transdisciplinario — de, para y en la vida — como otra forma de hacer y de producir conocimiento. En este artículo se verá como alternativa a qué tipo de conocimiento se desarrolla la bioética, caracterizando en primer lugar el paradigma científico moderno, de cuya "crisis" nace la bioética. Este ensayo surge de un proceso de revisión bibliográfica, intercambio de ideas y análisis, durante el curso de ética y ciencia que se imparte en el posgrado de Estudios Sociales de la Ciencia, en el Instituto Venezolano de Investigaciones Científicas.

Palabras clave: ciencia, bioética, ética, política, transdisciplinariedad

\section{Science, ethics and politics: bioethics as a way for transforming scientific praxis}

\begin{abstract}
Currently science is found in a paradoxical situation: the basis of scientific activity and the interrelations between science, technology, industry, society and State are strongly questioned and criticized, at the same time that the worldly idea of science as the only motor for human development prevails. There is where the idea of bioethics arises as trans-disciplinary knowledge - for, to and from life - as another way of doing and producing knowledge. In this article, the development of bioethics will be seen as an alternative to a type of knowledge, characterized in the first place as modern scientific paradigm, from which crisis, bioethics is born. This essay arises from a process of bibliographical review, exchange of ideas and analysis, during the course of ethics and science given as post-degree in Social Studies, in the Venezuelan Institute of Scientific Research.
\end{abstract}

Key words: science, bioethics, ethics, politics, trans-discipline

Ciência, ética e política: a bioética como caminho para a transformação da práxis científica

Resumo: A "ciência” se encontra hoje em dia numa situação paradoxal: os fundamentos da atividade científica e das interrelaçôes entre ciência, técnica, indústria, sociedade e Estado são fortemente questionados e criticados, ao mesmo tempo em que segue prevalecendo mundialmente a ideia de que a ciência constitui o único motor para o desenvolvimento humano. É onde surge a ideia da bioética como conhecimento transdisciplinar — de, para e na vida - como outra forma de fazer e de produzir conhecimento. Neste artigo se verá como alternativa que tipo de conhecimento desenvolve a bioética, caracterizando em primeiro lugar o paradigma científico moderno, de cuja "crise" nasce a bioética. Este ensaio surge de um processo de revisão bibliográfica, intercâmbio de ideias e análise, durante o curso de ética e ciência que se leciona na pós-graduação de Estudos Sociais da Ciência, no Instituto Venezuelano de Investigaçôes Científicas.

Palavras-chave: ciência, bioética, ética, política, transdisciplinaridade

\footnotetext{
${ }^{1}$ Reedición autorizada del artículo publicado en Revista de Bioética Latinoamericana 2014; 13: 47-64.

${ }^{2}$ Centro de Estudio de Transformaciones Sociales, Ciencia y Conocimientos, Instituto Venezolano de Investigaciones Científicas, Venezuela Correspondencia: xigon@gmail.com
} 


\section{Introducción}

La visión de la ciencia como objetiva, racional y motivada únicamente por la búsqueda y el amor a la "verdad" ha instaurado durante mucho tiempo la disyunción entre saber y ética. Esta manera de concebir la actividad científica, fundada en la clásica dicotomía entre "hecho" y "valor" o, dicho en otras palabras, entre el "ser" y el "deber ser", hizo de la ciencia una actividad supuestamente desinteresada y neutral, cuyo único y universal valor sería la verdad.

De ahí la ciega creencia en los infinitos beneficios que la ciencia traería a la humanidad y que se resume en la idea misma de "progreso", plasmada luego en la idea de "desarrollo". Sin embargo, el modelo de desarrollo científico-tecnológico, tributario de esta conceptualización del ser y del hacer de la ciencia, es hoy en día fuertemente cuestionado por la preocupante situación medioambiental, los diferentes problemas ligados al calentamiento global, y, entre muchos otros temas, la utilización de las armas químicas en muchos conflictos bélicos.

La "ciencia" se encuentra hoy en una situación paradójica: los fundamentos mismos de la actividad científica y de las interrelaciones entre ciencia, técnica, industria, sociedad y Estado son fuertemente cuestionados y criticados, al mismo tiempo que sigue prevaleciendo mundialmente la idea de que la ciencia constituye el único motor para el desarrollo humano.

El problema radica en el hecho de que tanto la "naturaleza" como el "hombre" no pueden ser reducidos a "objetos" de la ciencia, sin que se planteen una serie de problemas sociopolíticos y éticos que la misma ciencia, tal como está pensada, estructurada, desarrollada y aplicada, no está en capacidad de resolver. ¿Cómo pensar y hacer otra ciencia sin, por lo tanto, rechazar o desechar la idea misma de "ciencia"?

Ahí es donde surge la bioética ${ }^{3}$, como conoci-

\footnotetext{
${ }^{3}$ Según la definición de Van Rensselaer Potter (1971), se define justamente como un conocimiento cuya finalidad está orientada a saber cómo usar el conocimiento científico, de manera de preservar el bien social y la supervivencia de la vida en el planeta. Se trata por ende de subrayar la necesidad de acompañar el progreso científico de una reflexión ética que tome en cuenta los valores y la "totalidad" (la sociedad, la naturaleza, la biosfera).
}

miento transdisciplinario, de, para y en la vida, como otra forma de hacer y de producir conocimiento. Es decir, como actividad cognitiva que, al romper con la oposición entre sujeto y objeto, entre hecho y valor, no privilegia únicamente y exclusivamente el cómo funcionan las cosas, sino que integra tanto el agente de ese conocimiento (la subjetividad) como el fin del mismo (el telos). Si bien en su acepción mas común la bioética es reducida a sus dimensiones prácticas (comités de bioética) y normativas (códigos de bioética), y asimilada al ámbito exclusivo de la clínica, es decir a la resolución de asuntos exclusivamente relacionados con las ciencias de la salud, en su acepción "integral" se presenta como una reflexión que va tomando impulso en el estudio de las consecuencias del modelo de desarrollo científico tecnológico para y sobre la vida en general, y desarrollando una reflexión critica sobre la idea misma de ciencia que sustenta dicho modelo.

Es así como se puede ver en la bioética un espacio en el cual se plantea no solo el desarrollo de un cierto tipo de conocimiento, que permita medir, corregir, prevenir o regular las consecuencias del desarrollo científico-tecnológico sobre el ser humano, las sociedades, la naturaleza o la biósfera, sino también como una reflexión profunda y holística sobre el acto de conocer, que implica asumir la medida de las formas problemáticas y cuestionables que ha tomado el desarrollo del conocimiento científico-tecnológico para, desde ahí, desplegarse como un campo de estudio práctico, implicando otra forma de hacer ciencia, otra forma de realizar el acto del conocer, basado en la transdisciplinariedad y en la intercomunicación.

Para entender como alternativa a qué tipo de conocimiento se desarrolla la bioética, es necesario categorizar en primer lugar el paradigma científico moderno, de manera de entender cómo es la estructura misma de este tipo de conocimiento que lleva a su crisis parcial. Crisis de la cual nace, justamente, la bioética.

\section{Categorizaciones y limitaciones del paradigma científico moderno}

De manera sintética, ¿cuáles son las rasgos principales con que podemos definir la ciencia moderna?(1). Primero que todo, esta se constituye 
en cuanto conocimiento racional positivista (reducción de lo real a lo racional), determinista (predominio de la búsqueda de la causa formal) e instrumental (dominación de la naturaleza por la técnica).

La primera gran reducción, la positivista ${ }^{4}$, se establece como compendio de lo real a lo que la razón, a través la aplicación del método científico, puede explicar. En este orden de ideas, la realidad está constituida únicamente por lo que la razón humana puede conocer. Este conocimiento positivista se desarrolla a través la implementación de metodologías racionales de aprehensión de la realidad, tales como la experimentación, la deducción-inducción y la demostración, que confieren a este tipo de conocimiento su carácter "objetivo". De ahí que se considere como conocimiento solo lo que puede ser desarrollado a través de estos métodos, que descalifican cualquier otra forma que no corresponda a esta definición de objetividad. A esta primera gran reducción corresponde la separación entre el objeto-objetividad (científica) y el sujeto-subjetividad (no científica).

De esta idea se desprende la caracterización de la finalidad del conocimiento científico: la supuesta búsqueda desinteresada de la "verdad". La verdad es pensada únicamente como lo que puede ser determinado a través de métodos de análisis y de cálculo, métodos que supuestamente conceden a esa verdad su carácter de universalidad, desprendida del espacio y del tiempo, es decir, de sus condiciones de producción. La verdad es entonces esa gran quimera que la razón científica se esfuerza por alcanzar, en el marco, justamente, de la reducción de lo real a lo que puede ser científicamente conocido.

La segunda gran reducción, ligada a la primera, se sustenta en el carácter determinista del conocimiento científico, exclusivamente volcado a la búsqueda de la o las causa(s) formal(es) de las cosas, es decir en el cómo se hace o en el cómo suce$\mathrm{de}^{5}$. Aquí es donde el sujeto, en cuanto agente de este conocimiento, es definitivamente excluido y

${ }^{4}$ El método de Descartes representa una ilustración acabada de estos métodos racionales positivistas, si bien el término de "positivismo" fue utilizado por primera vez por Comte.

${ }^{5}$ El carácter determinista de la ciencia puede verse como una reducción de la definición aristotélica de la causa a la única causa formal, dejando de lado el estudio de la causa final. expulsado del conocimiento científico. La objetividad del conocimiento científico solo puede darse si se elimina todo lo que pudiese contaminar o interferir en la búsqueda de la causa, es decir, en la determinación de la relación causa-efecto o causa-consecuencia. El determinismo, que radica en la elaboración de leyes como método de aprehensión de la realidad, excluye entonces la idea de finalidad (telos) y, por lo tanto, la idea de que el conocimiento pudiese integrar en sus problemáticas el fin de las cosas.

Esta segunda gran reducción es la que impide a la ciencia toda real posibilidad de reflexividad(2): si la ciencia no se preocupa por el telos o, dicho de otra forma, si el telos no solo no puede ser objeto de conocimiento científico, sino que no tiene pertinencia en la elaboración del conocimiento científico, entonces la finalidad o el por qué de la ciencia deja de ser objeto de reflexión. La ciencia se despliega entonces como conocimiento cerrado sobre sí mismo: la finalidad de la ciencia es únicamente la búsqueda de la verdad, verdad que solo puede ser alcanzada a través del conocimiento científico y de la elaboración de teorías, leyes y sistemas, que impiden totalmente considerar la complejidad de la realidad de la cual este mismo conocimiento científico hace parte.

Ahí es donde se encuentra el nudo de la segunda gran distinción que la ciencia elabora entre el hecho (objeto de conocimiento científico) y el valor (objeto de creencia), o entre el conocimiento y la opinión6. Distinción que expulsa entonces también toda idea de ética, de responsabilidad o de sentido común(3), y que pretende hacer de la ciencia un conocimiento neutral y por ende desinteresado. El gran mito de la ciencia como conocimiento altruista, fundado en un amor puro y desinteresado por la verdad, esconde entonces la tercera gran reducción que radica en su carácter instrumental.

La técnica es el instrumento privilegiado de la ciencia para la manipulación de la naturaleza en

\footnotetext{
${ }^{6}$ Obviamente, esta distinción es heredera directa de la clásica distinción griega entre episteme, es decir, saber o conocimiento, y doxa, opinión. Sin embargo, esta distinción está en la base de la expulsión propiamente moderna de las consideraciones éticas o morales del campo de la ciencia.
} 
vista de su dominación? ? Dominación idealizada en la idea de "progreso", justificación ideológica para todas las manipulaciones científicas, tanto de la naturaleza como del hombre y de las sociedades, plasmada luego en la idea de "desarrollo", justificación para la dominación militar e industrial del mundo.

En efecto, la tecnificación de la ciencia como instrumento de dominación de la naturaleza, y por ende del ser humano y de la sociedad, transforma el instrumento (la técnica) en el fin último del desarrollo científico. La relación de la ciencia moderna con la naturaleza, mera objetivización, hace de esta un simple instrumento al servicio del desarrollo de su dominación sobre el ser humano.

Las consecuencias de estas tres reducciones son, primero que todo, que la ciencia, que pretende universalidad, termina - al contrario, en cuanto unidimensiona la realidad - siendo pura especialización y fragmentación. En efecto, la ciencia, en su modo de organización, ha llegado a un tal grado de especialización que la intercomunicación en su propio seno se hace muchas veces imposible, desembocando en una fragmentación de los conocimientos especializados, incapaces de dialogar entre ellos.

Segundo, que en su afán por reducirse a la búsqueda objetiva de las causas, se vacía de toda significación, dejando de lado las grandes cuestiones relativas al sentido de la vida, de la sociedad, del mundo o de la naturaleza. Y tercero que, en su dimensión instrumentalista, concluye obviando los grandes problemas que ella misma desencadena, tales como la amenaza, posibilidad o realidad de destrucción parcial o total del planeta y de la humanidad, y desentendiéndose de las consecuencias de un modelo de desarrollo científico tecnológico que no cumple con ninguna de sus promesas de progreso o de desarrollo, dejándonos un mundo donde predomina la pobreza, la exclusión, el hambre, la enfermedad y la guerra.

\footnotetext{
${ }^{7}$ Tal como lo afirma Weber, la ciencia permite darnos "una respuesta a la pregunta: ¿qué debemos hacer si queremos dominar técnicamente la vida? Pero en cuanto a responder si todo esto tiene en el fondo algún sentido o preguntarse por qué debemos o queremos ser dueños técnicamente de la vida lo dejan totalmente en suspenso o bien lo presuponen para sus propios fines".
}

Por su propia estructura y finalidad, la ciencia moderna desencadena entonces incertidumbre, ambigüedad e incomprensible complejidad. En pocas palabras, desemboca en una generalizada desposesión cognitiva que nos deja totalmente indefensos frente a la complejidad del mundo y nuestras acciones en él.

\section{Ciencia, política y ética}

Se plantean entonces una serie de problemas que podríamos resumir mediante las siguientes preguntas:

- La ciencia, ¿no presupone imperativos fundados en valores que no se reducen únicamente al concepto de "verdad"?

- La dogmatización misma del concepto de verdad, ¿no presupone a su vez una cierta concepción del ser humano en términos de valores?

- La ciencia, ¿puede realmente ser independiente de la utilización que se hace de ella y de los valores implicados en dicha utilización?

- Conceptos como "verdad", "objetividad" y "racionalidad", ¿no pueden ser vistos como producto de una cierta "ideología de la ciencia”, lo que cuestionaría su supuesta neutralidad ética?

- ¿Pueden realmente separarse la ciencia y la técnica, es decir, el conocimiento y uso del conocimiento?

La bioética, en cuanto práctica o praxis cognitiva, se plantea justamente como espacio para el desarrollo crítico de estos temas, a partir de la articulación efectiva entre ciencia, política y ética.

Para poder plantear esta interacción ciencia-política-ética, es necesario reinsertar la ciencia en su entorno, es decir plantearla como campo social, o, dicho de otro modo, reinsertar la ciencia en el contexto sociopolítico de su propia producción, es decir, reconocer el nexo entre ciencia y política.

La ciencia no es una actividad neutra, sino que obedece a una serie de intereses que se esmera en disimular. Tal como lo define Bourdieu(4), el lla- 
mado "desinterés del científico" no es otra cosa que un "interés especifico": la acumulación del capital científico-tecnológico en vista de la reproducción del sistema científico-tecnológico de dominación de nuestra representación del mundo y, por ende, de dominación del mundo.

La institucionalización e industrialización modernas de la ciencia, plasmadas en los modelos desarrollistas, han llevado a la implementación de un modelo de organización y de producción científica que nada tiene de desinteresado, ni por sus relaciones directas o indirectas con el complejo militar-técnico-industrial, ni por su estratificación estructuralmente capitalista, identificable en su afán autorreproductor de acumulación de capital y de monopolio de autoridad científica. La comunidad científica es, en cuanto tal, una comunidad política sumamente estratificada y jerarquizada, que sustenta los principales modelos de dominación industrial-socio-político hegemónicos mundiales. La ciencia es un modelo de explicación, de producción y de reproducción de una cierta concepción.

La ciencia en cuanto ideología, tal como Habermas(5) la define, se desarrolla entonces como dominación técnica instrumental (por el predominio de la razón técnica), regulando y controlando nuestra aprehensión de la naturaleza, del hombre y la sociedad, y nuestro modo de relacionarnos con estos. La ciencia se constituye como un verdadero modelo de dominación operativo, es decir como poder "sobre", como poder ilimitado.

La ciencia en cuanto ideología tiene entonces como finalidad justificar este poder ilimitado: a través la dogmatización de la libertad científica (entendida como ausencia de limitación de su poderío y campo de acción), que se justifica a posteriori como instrumento necesario del progreso y del desarrollo, que llevan a la humanidad a la máxima felicidad técnica (confort). Nos encontramos entonces con la trilogía: libertad-progresofelicidad.

Sin embargo, la ciencia que revindica su poder ilimitado se autorrepresenta negando su dimensión política: la libertad científica pretende ser una libertad neutra y desinteresada.
Aquí entra en juego la primera paradoja: se ha venido superponiendo a esta caracterización del poder ilimitado de la ciencia, supuestamente apolítico y neutral, la conciencia de la existencia de un descontrol sobre este poder, es decir, de una imposibilidad de dominar la dominación.

En efecto, esta paradoja principal puede ser resumida de la siguiente forma: la ciencia se constituye como un poder absoluto (sobre el ser humano, la naturaleza, la sociedad) que no tiene poder alguno sobre sí mismo $(3,6)$. Y no lo tiene porque no puede ser autorreflexiva. El hecho de que la ciencia se piense como neutralidad y reivindique su desvinculación con toda dimensión política, hace imposible que pueda asumir control reflexivo sobre ella misma, ya que todo control es visto como una injerencia externa de la política sobre la ciencia. La ciencia moderna propicia entonces a lo interno una irresponsabilidad generalizada, en la cual la ciencia es presentada como pura y neutra, la técnica como buena o mala, según el caso, y la política como la causante de todos los males(3).

Los científicos, encerrados en sus especialidades, que los desvinculan de todo problema que no haya sido recortado y reducido a la pequeña parcela de conocimiento que poseen y defienden, no se sienten responsables o corresponsables en el acontecer del mundo. La ciencia, que ha perdido su fe en la idea de progreso, que ya no cree siquiera en los modelos desarrollistas que propicia, no tiene ningún instrumento que le permita entrar en relación con la realidad que ella misma transforma, y por ende actuar sobre estas transformaciones.

La ciencia está encerrada y aislada en su fragmentación disciplinaria y disciplinada, y se lava las manos, con grados diferentes de mala conciencia y mala fe, respecto de cualquier idea de ética o de responsabilidad. De tal manera, se condena ella misma al inmovilismo, otro nombre de la impotencia. Es justamente a partir de la idea de responsabilidad que la bioética, en cuanto praxis cognitiva, toma impulso.

\section{La bioética como praxis emancipadora}

En este contexto, la bioética se plantea como praxis cognitiva emancipadora, pero, ¿̇emancipación 
de qué? La ciencia moderna hace o desencadena muchas cosas y fenómenos sobre los cuales no tiene poder o control. Y si el saber es poder, y poder es poder de hacer, entonces el reto más grande que enfrenta la ciencia moderna es reelaborarse en cuanto saber del hacer, para salir así de su impotencia, rompiendo las cadenas de su desposesión cognitiva.

Sobre y en contra de esta desposesión cognitiva, De Sousa Santos desarrolla lo que llama una "epistemología del Sur":

"Entiendo como epistemología del Sur la búsqueda de conocimientos y de criterios de validez del conocimiento que otorguen visibilidad y credibilidad a las prácticas cognitivas de las clases, de los pueblos y de los grupos sociales que han sido históricamente victimizados, explotados y oprimidos por el colonialismo y el capitalismo globales. (...) no habrá justicia social global sin justicia cognitiva global.

Los procesos de opresión y de explotación, al excluir grupos y prácticas sociales, excluyen también los conocimientos usados por esos grupos para llevar a cabo esas prácticas. A esta dimensión de la exclusión la he llamado epistemicidio. La epistemología del Sur, al mismo tiempo que denuncia el epistemicidio, ofrece instrumentos analíticos que permiten, no solo recuperar conocimientos suprimidos o marginalizados, sino también identificar las condiciones que tornen posible construir nuevos conocimientos de resistencia y de producción de alternativas al capitalismo y al colonialismo globales. En esto consiste la propuesta de una ecología de los saberes"(1:12).

He aquí el primer punto sobre le cual la bioética se aplica: la desposesión cognitiva solo podrá desarticularse asumiendo la dimensión política y ética de la ciencia en su relación con lo que no es ciencia, es decir, en relación con la sociedad, con lo otro y los otros. ¿Y qué es ese otro? Lo que no es ciencia o solo es objeto de esta: la naturaleza, la sociedad, la subjetividad. La bioética global o integral plantea una reestructuración de la ciencia a partir de la integración de la subjetividad dentro de su campo de definición y acción. El "epistemicidio" es entonces la destrucción de la otredad, de la subjetividad, de todo conocimiento que no sea considerado racional.
La bioética nace a partir de la identificación de la paradoja del impoder de la ciencia sobre su propio poder, planteándose como tarea anticipar y pensar, para desarticularlas, las consecuencias de este impoder (destrucción de la naturaleza, del planeta, de la humanidad). Es decir, su tarea principal es responsabilizar a la ciencia respecto de las consecuencias del progreso y del desarrollo científico tecnológico.

Pero, no se trata de enjuiciarla ni de descalificarla en cuanto tipo de conocimiento válido, sino de transformarla. La primera tarea en este plan de transformación consiste entonces en reubicar la ciencia en sus dimensiones sociopolíticas, para justamente transformar estas relaciones.

La bioética, como praxis cognitiva emancipadora, plantea dos ejes principales:

Primero, el estudio de las dimensiones sociopolíticas de la ciencia. Para ello es necesario, sin descartar los conocimientos científicos parcializados, desarrollar otras formas de conocimiento e integrar los tipos y formas existentes a partir de la articulación e interpenetración entre estos. El paradigma de la transdisciplinariedad nace justamente de la necesidad de estudiar la realidad de manera integral, en sus diferentes facetas, incorporando la dimensión de finalidad y refundando de tal manera el conocimiento científico.

Es en la dimensión disciplinaria de la ciencia que se encuentra la raíz de la fragmentación del conocimiento científico, así como del epistemicidio (destrucción o invalidación de otros conocimientos), que le hace imposible considerar su propia finalidad y por ende las consecuencias de su desarrollo. La tarea fundamental que se plantea la bioética en su reflexión sobre el acto de conocimiento es, entonces, romper con la compartimentación de la ciencia que estructura su modo de relación excluyente con el entorno que la condiciona, separándola de este.

La idea motriz de la bioética es la de desarrollar un conocimiento holístico para posicionarse como conocimiento de la vida (bio) y no sobre parcelas compartimentadas de esta; un tipo de conocimiento que integra la vez el telos y la subjetividad, es decir, que se aplique a inter-conocer 
y trans-conocer la realidad en todas sus dimensiones. No se trata de un conocimiento acumulativo ni de otro catálogo de la realidad, sino de una praxis cognitiva que se construye dentro y como intercomunicación entre las diferentes facetas de la realidad vivida, de manera de desarrollar una verdadera ecología de los conocimientos. De ahí la idea de pensamiento complejo, desarrollada por $\operatorname{Morin}(7)$, que plantea justamente interconectar distintas dimensiones de lo real que hacen su complejidad, su multidimensionalidad ${ }^{8}$.

La transdisciplinariedad no es entonces otra disciplina, sino una postura cognitiva, una praxis que integra la intersubjetividad y la construcción co-

\footnotetext{
${ }^{8}$ Para Morin (1998), hay tres grandes principios que definen esta complejidad. En el principio dialógico se asocian dos términos antagónicos para hacerlos dialogar y de cierta forma mantener la dualidad en el seno de la unidad. Aquí el punto es mantener las paradojas de las cuales solo puede aparecer la coherencia. El principio de recursividad, por su parte, rompe con el predominio de concepto de causalidad, ya que son recursivos los productos y los efectos, que son, al mismo tiempo, causas y productores de aquello que los produce. Ahí encontramos la idea central de interacción. Finalmente el principio de hologramía, que transciende el reduccionismo, en el cual la parte está en el todo y, especialmente, el todo en la parte. Los principales conceptos del pensamiento complejo son así los de distinción, conjunción e implicación.
}

lectiva del conocimiento. Se trata —al contrario del conocimiento científico moderno que diseca la vida - de pensar la realidad hecha vida.

El segundo eje de la bioética es hacer del conocimiento una herramienta de transformación de la realidad, pero no a partir de la idea de dominación, sino de la idea de responsabilidad y de corresponsabilidad. He ahí el carácter ético de la bioética. El saber al cual aspira la bioética es un saber hacer, una práctica que se responsabiliza como saber del hacer que desarrolla.

La bioética se posiciona entonces como postura que no vacila en asumir la acción humana. Esta se plantea como conocimiento-acción y, en cuanto tal, como ética, es decir, como praxis cognitiva responsable de sí misma. De ahí la idea de emancipación. La dimensión ética de la bioética es lo que transforma el saber en un saber del hacer haciendo, liberando los conocimientos sometidos, integrando el sujeto y las subjetividades, celebrando la inter y transcomunicación, el hecho como valor y haciendo del saber un poder, es decir un poder hacer, como saber del poder hacer de la vida.

\section{Referencias}

1. De Sousa Santos B. Una Epistemología del Sur. La reinvención del conocimiento y la emancipación social. México: Clacso; 2009.

2. Husserl E. Crisis de las ciencias europeas y la fenomenología trascendental: una introducción a la filosofía fenomenológica. Barcelona: Crítica; 1991.

3. Morin E. Ciencia con Conciencia. Barcelona: Editorial Anthropos; 2006.

4. Bourdieu P. Los usos sociales de la ciencia. Buenos Aires: Ediciones Nueva Visión; 2003.

5. Habermas J. Ciencia y técnica como ideología. Madrid: Ed. Tecnos; 1986.

6. Jonas H. El principio de responsabilidad. Ensayo de una ética para la civilización tecnológica. Barcelona: Herder; 1975.

7. Morin E. Introducción al pensamiento complejo. Barcelona: Gedisa Editorial; 1998.

Recibido: 21 de febrero de 2013

Aceptado: 19 de mayo de 2013 